\title{
Laminite endocrinopática em equinos com síndrome metabólica: características clínicas, tratamento e evolução em três pacientes - relato de caso
}

\author{
[Endocrinopathic laminitis in horses with metabolic syndrome: clinical characteristics, \\ treatment and evolution in three patients - case report] \\ L.A.O. Paula ${ }^{1}$, K.R.J.L. Lera ${ }^{2}$, B.R.F. Schuh ${ }^{1}$, F.F.A. Silva ${ }^{1}$, \\ E. Michelon do Nascimento ${ }^{1}$, G.M. Pagliosa ${ }^{3}$ \\ ${ }^{1}$ Aluno de pós-graduação - Universidade Federal do Paraná - Palotina, PR \\ ${ }^{2}$ Aluno de pós-graduação - Universidade Estadual Paulista - Jaboticabal, SP \\ ${ }^{3}$ Universidade Federal do Paraná - Palotina, PR
}

\begin{abstract}
RESUMO
Laminite endocrinopática designa os casos de laminite cuja etiologia está associada a uma endocrinopatia. Cavalos com síndrome metabólica equina (SME) apresentam adiposidade regional e obesidade. Existe uma correlação positiva entre obesidade e resistência à insulina. Este relato descreve três casos de laminite endocrinopática em cavalos com SME. Os pacientes apresentaram escore de condição corporal (ECC) variando de 8 a 9 (escala de 1 a 9), sensibilidade ao teste de pinçamento do casco e claudicação de grau 3 ou 4 (escala de 1 a 4). Não havia histórico de cólica recente, trauma ou excesso de exercício. $\mathrm{O}$ tratamento incluiu medicação anti-inflamatória não esteroidal, repouso na baia, restrição energética para perda de peso, revestimento e bandagem dos membros, conforme a necessidade de cada paciente. A restrição alimentar ajudou no tratamento da SME e reduziu o ECC. Tratamento medicamentoso, casqueamento e bandagem diminuíram o grau de claudicação. Conclui-se que o tratamento antiinflamatório não esteroidal, a restrição energética, o repouso em baia, o casqueamento e a bandagem do casco são eficazes no tratamento da laminite endocrinopática em cavalos com síndrome metabólica equina.
\end{abstract}

Palavras-chave: adiposidade regional, cavalos, claudicação, obesidade

\begin{abstract}
Endocrinopathic laminitis refers to cases of laminitis whose etiology is associated with an endocrinopathy. Horses with Equine Metabolic Syndrome (EMS) have regional adiposity and obesity. There is a positive correlation between obesity and insulin resistance. This report describes three cases of endocrinopathic laminitis in horses with EMS. Patients had body condition score (BCS) ranging from 8 to 9 (scale from 1 to 9), sensitivity to the hoof clamping test and claudication grade 3 or 4 (scale from 1 to 4). There was no history of recent colic, trauma or over exercise. Treatment included non-steroidal anti-inflammatory medication, stall rest, energy restriction for weight loss, hoofing and hoof bandage as needed by each patient. Dietary restriction helped in the treatment of EMS and reduced BCS. Drug treatment, hoofing and hoof bandage decreased the degree of lameness. It is concluded that non-steroidal anti-inflammatory treatment, energy restriction, stall rest, hoofing and hoof bandage are effective in the treatment of endocrinopathic laminitis in horses with equine metabolic syndrome.
\end{abstract}

Keywords: regional adiposity, horses, lameness, obesity

\section{INTRODUÇÃO}

A laminite em equinos é uma afecção grave, de prevalência alta, reconhecida como uma das mais importantes síndromes clínicas na espécie. Entre

Recebido em 13 de novembro de 2019

Aceito em 10 de março de 2020

E-mail: liliane.op@gmail.com suas causas estão afecções primárias associadas à sepse e endotoxemia, como cólica, metrite entre outras; excesso de peso sobre um membro e endocrinopatias (Silva et al., 2013). O termo laminite endocrinopática foi adotado para classificar os casos de laminite em que a origem 
está mais fortemente associada a uma endocrinopatia subjacente, como a resistência à insulina (RI) decorrente da síndrome metabólica equina, influência de corticosteroides e síndrome de Cushing (Frank, et al., 2010; Johnson et al., 2010).

A síndrome metabólica equina (SME) é semelhante à síndrome metabólica (SM) em humanos, que é uma coleção de fatores de risco avaliados para prever a ocorrência de doença arterial coronariana e diabetes mellitus tipo $2 \mathrm{em}$ pessoas. As características corporais que definem animais afetados pela SME são a obesidade e a adiposidade ao redor do ligamento nucal na região cervical (crista do pescoço), na base da cauda, na região escapular, no prepúcio ou na glândula mamária (Frank et al., 2010).

A correlação entre a obesidade e a resistência à insulina é a liberação de citocinas inflamatórias pelos tecidos adiposos. Em indivíduos obesos, altas concentrações de citocinas são excretadas para além dos tecidos de origem; as citocinas inibem a sinalização do receptor de insulina, levando à redução da sensibilidade à insulina (Paz et al., 2013; Tadros e Frank, 2013). O objetivo deste trabalho foi relatar três casos de laminite endocrinopática, destacando sua evolução clínica, terapêutica e manejo complementar específico.

\section{CASUÍSTICA}

Equino 1: macho, 19 anos de idade, sem raça definida, utilizado para montaria, com claudicação intermitente em membro pélvico esquerdo há cerca de dois anos, sem histórico de cólica, trauma ou exercício intenso. A dieta era baseada em aveia $1,5 \mathrm{~kg}$ em duas refeições, feno de tifton, sal mineral e água à vontade.

No exame clínico, observou-se escore de condição corporal (ECC) de 9 na escala de Henneke et al. (1983), com depósito de gordura na crista do pescoço e na base da cauda (Fig. 1A), frequência cardíaca (FC) de $60 \mathrm{bpm}$ (batimentos por minuto) e termometria de $38,7^{\circ} \mathrm{C}$. Verificou-se, também, claudicação de grau 3 em escala de Obel (1948), pulso digital de intensidade moderada, nos membros torácico e pélvico esquerdos (MTE e MPE), e forte, nos membros torácico e pélvico direitos (MTD e MPD), presença de anéis divergentes na muralha do casco de todos os membros, com sensibilidade ao pinçamento na sola em região de pinça em todos os cascos e fístula na região de coroa do casco do MTD, com drenagem de coloração escura e odor fétido. Com base no histórico e achados clínicos, o diagnóstico foi de laminite endocrinopática.

O tratamento orientado foi $1,1 \mathrm{mg} / \mathrm{kg}$ de flunixina meglumina, a cada $24 \mathrm{~h}$ (SID), e $0,01 \mathrm{mg} / \mathrm{kg}$ de acepromazina, a cada $12 \mathrm{~h}$ (BID), por 15 dias, além de crioterapia nos cascos por oito horas por dia, sendo quatro horas durante a manhã e quatro horas durante a tarde, repouso em baia com boa forragem de maravalha e retirada do concentrado da dieta.

Após 15 dias de tratamento, o grau de claudicação foi reduzido a 2, mas o paciente foi internado no Hospital Veterinário do Setor Palotina da Universidade Federal do Paraná (HVP - UFPR) devido à presença de fístulas na região de coroa do casco nos membros pélvicos e na pinça dos cascos dos membros torácicos. No casqueamento constatou-se que o estojo córneo estava friável (Fig. 1B), o casco estava delgado na região de pinça na sola de todos os membros e havia discreta secreção escura e fétida. O exame radiográfico dos cascos revelou rotação palmar de $11,2^{\circ}$ da terceira falange (Fig. 1C). Realizouse antissepsia com iodopolividona, aplicação de tintura alcoólica de iodo a $10 \%$ nas fístulas na sola e bandagem com algodão hidrofóbico e atadura de crepe em todos os cascos. Esse curativo foi repetido duas vezes por semana, nas primeiras seis semanas, e depois semanalmente, até o final do tratamento.

A terapia anti-inflamatória foi mantida por mais 30 dias, acrescida de omeprazol $(2 \mathrm{mg} / \mathrm{kg}$, SID, VO), biotina $(50 \mathrm{mg} / \mathrm{kg}$, SID, VO) e, devido à secreção da sola, prescreveu-se ceftiofur $(2,2 \mathrm{mg} / \mathrm{kg}, \mathrm{SID}, \mathrm{IM}$, três dias). Manteve-se o repouso em baia e foram adicionados tapetes de borracha sob a maravalha. Realizou-se casqueamento a cada 21 dias e a crioterapia foi interrompida. Instituiu-se dieta com restrição energética para perda de peso, com feno $1 \%$ do peso corporal, dividida em seis refeições diárias, e manteve-se o sal mineral e a água à vontade. No sexto mês de internação, o paciente recebeu alta, com ECC de 7; sem claudicação, sem sensibilidade ao pinçamento dos cascos nem pulso digital palpável; o estojo córneo tinha 
consistência normal e as fístulas tinham fechado. O proprietário foi orientado a controlar o peso do paciente e a retomar o exercício gradualmente.

Aproximadamente dois anos após o primeiro atendimento, o paciente foi encaminhado ao HVP - UFPR em duas ocasiões, com intervalo de 60 dias entre elas, por motivo não relacionado a sistema locomotor. Nessas duas ocasiões, observou-se que o paciente apresentava ECC 9, claudicação de grau $2 \mathrm{e}$, mesmo sem pulso digital palpável, foi observada dor ao pinçamento na região de pinça na sola dos quatro cascos, presença de hematomas na região lateral da muralha e em pontos da sola, em todos os cascos, caracterizando laminite endocrinopática. Prescreveu-se fenilbutazona $(4,4 \mathrm{mg} / \mathrm{kg}$, SID, IV) nos três primeiros dias, seguido de meloxicam $(0,6 \mathrm{mg} / \mathrm{kg}, \quad$ SID, IM) e acepromazina $(0,01 \mathrm{mg} / \mathrm{kg}$, BID, IM) por 15 dias, além de repouso em baia com cama macia e restrição energética para perda de peso. Após 15 dias, houve melhora na claudicação e na sensibilidade ao pinçamento. O paciente obteve alta e foi recomendado ao proprietário manter a dieta para perda de peso.

Equino 2: fêmea, sem raça definida, de 12 anos de idade que há cerca de cinco meses antes do atendimento no hospital era exercitada na guia duas vezes por semana, por 30 minutos. Não havia histórico de cólica ou trauma. A dieta consistia em $1 \mathrm{~kg}$ de aveia em duas refeições, feno de tifton, água e sal à vontade. Devido à claudicação dos membros torácicos, a paciente já havia sido afastada do treinamento e recebido crioterapia nos cascos, mas não apresentou melhora clínica.

O exame clínico revelou a presença de anéis divergentes na muralha do casco nos quatro membros (Fig. 1D), sensibilidade ao pinçamento da sola na região de pinça dos membros torácicos e aumento de temperatura na região de coroa do casco dos quatro membros. A paciente exibia ECC de 9, com depósito de gordura na crista do pescoço, nos glúteos e na base da cauda. Com base nos achados clínicos, o diagnóstico foi de laminite endocrinopática.

O tratamento incluiu flunixina meglumina $(1,1 \mathrm{mg} / \mathrm{kg}, \mathrm{SID}, \mathrm{IM})$, acepromazina $(0,01 \mathrm{mg} / \mathrm{kg}$, BID, IM) e omeprazol (2mg/kg, SID, VO), ambos por 30 dias. Durante o internamento, a paciente foi mantida em baia forrada com tapetes de borracha e cama de maravalha. Foi realizado casqueamento quinzenal e, como o casco estava friável e apresentava fístula na região de coroa, recebeu bandagem. Preconizou-se dieta com redução de energia, baseada na oferta de feno (1\% peso corporal), dividida em seis refeições ao longo do dia, sal mineral e água à vontade. Após cinco meses de internamento, a paciente recebeu alta, com remissão dos sinais clínicos e ECC de 7.

Equino 3: fêmea da raça Crioula, de três anos, adquirida há 30 dias e treinada para laço. Após ser transportada por $100 \mathrm{~km}$ para um evento, a paciente manifestou claudicação grau 4 (Obel, 1948) de forma aguda, sem histórico de exercício intenso, trauma ou afecção prévia. A dieta consistia em $6 \mathrm{~kg}$ de ração, dividida em duas refeições, feno de tifton e água à vontade. O proprietário aplicou flunixina meglumina $(1,1 \mathrm{mg} / \mathrm{kg})$ três horas antes de encaminhar o animal para o HVP - UFPR. Findo o efeito antiinflamatório, a paciente parou de se alimentar, ficou em decúbito lateral e iniciou taquipneia. Ao ser estimulada a levantar-se, adquiriu postura antiálgica, caracterizada pelo desvio caudal do eixo de gravidade para transferir peso aos membros pélvicos e manteve claudicação grau 4.

Ao exame clínico, constatou-se ECC de 8, acúmulo de gordura na crista do pescoço e na base da cauda, FC 60bpm, FR 32mpm, termometria retal de $39,9^{\circ} \mathrm{C}$, presença de anéis divergentes na muralha, pulso forte na artéria digital e aumento de temperatura nos cascos em todos os membros. Ao exame radiográfico, verificou-se rotação palmar de $10,5^{\circ}$ da terceira falange (Fig. 1E). Devido aos achados clínicos e radiográficos, diagnosticou-se laminite endocrinopática.

O tratamento incluiu anti-inflamatóro não esteroidal (AINE), tendo sido empregado, nos três primeiros dias, fenilbutazona $(4,4 \mathrm{mg} / \mathrm{kg}$, SID, IV) e, posteriormente, firocoxibe $(0,1 \mathrm{mg} / \mathrm{kg}, \mathrm{SID}, \mathrm{VO})$, acepromazina $(0,01 \mathrm{mg} / \mathrm{kg}$, BID, IM) e omeprazol (2mg/kg, SID, VO), ambos por 90 dias. Retiraram-se apenas as ferraduras dos membros torácicos da paciente devido ao seu temperamento. Foi colocada bandagem elástica com espuma sintética vinílica acetinada (EVA) na sola do casco para aumentar o conforto da paciente, essa bandagem foi 
trocada semanalmente, até completar 30 dias. O intervalo entre casqueamentos foi de 30 dias. A paciente foi mantida em baia, sobre tapetes de borracha em cama de maravalha. Instituiu-se dieta com restrição energética à base de feno em $1,5 \%$ de peso corporal. Após três meses de internamento, a paciente recebeu alta, com grau de claudicação 1 e ECC de 7.

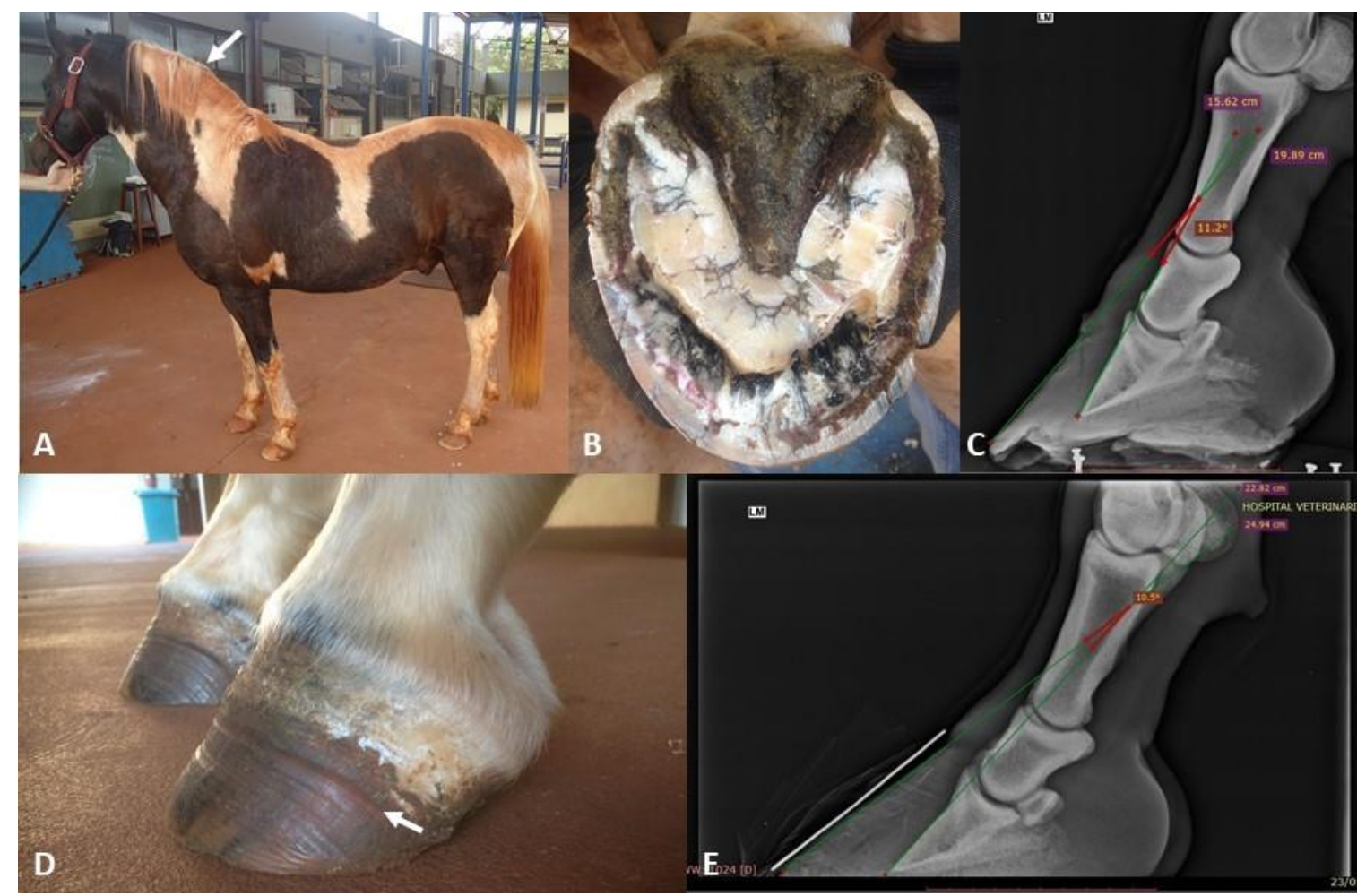

Figura 1. Laminite endocrinopática em equinos. A - adiposidade no pescoço (seta). B - casco de aspecto friável, com presença de hematomas e broca na sola. C - imagem radiográfica em projeção lateromedial do casco (MTE) do Equino 1 exibindo rotação palmar da falange distal. D - anéis divergentes na muralha do casco (seta). E - imagem radiográfica em projeção lateromedial do casco (MTD) do Equino 3 exibindo rotação palmar da falange distal.

\section{DISCUSSÃO}

Os equinos atendidos eram obesos e tinham adiposidade na crista do pescoço, na região glútea e na base da cauda, sinais característicos de síndrome metabólica equina (Frank et al., 2010). Esses achados, associados à claudicação bilateral intensa, à presença de pulso forte na artéria digital, ao aumento da temperatura dos cascos e à presença de anéis de crescimento divergentes no casco, permitem classificar o quadro clínico apresentado pelos pacientes como laminite endocrinopática (Silva et al., 2013). Segundo Menzies-Gow (2018), ainda não há um teste padrão-ouro para diagnóstico da afecção, porém é recomendado o teste de tolerância à insulina e o teste oral de glicose, além da radiografia lateromedial do membro, em caso de suspeita de rotação da falange distal.

A radiografia foi utilizada para avaliação do grau de rotação palmar/plantar da falange distal, que, segundo Stashak, quando superior a $5^{\circ}$, confirma o diagnóstico de laminite. Stick et al. (1982) sugerem que cavalos com rotação inferior a 5,5 têm melhor prognóstico para retorno às atividades atléticas do que cavalos com rotação superior a $11,5^{\circ}$. Os pacientes atendidos Equinos 1 e 3 apresentavam grau de rotação de $10,5^{\circ}$ e $11,02^{\circ}$, respectivamente; ambos retornaram as suas atividades, sendo o Equino 1 utilizado em atividade de equoterapia.

Muitos cavalos com SME podem manifestar laminite, e os pacientes relatados apresentaram a 
forma crônica da doença, pois apresentavam claudicação, dificuldade de deambulação e anéis na muralha dos cascos (Pollitt, 1999; Paz et al., 2013). Na forma aguda, essa afecção pode iniciar de forma espontânea, sem qualquer histórico de sobrecarga de grãos, infecção bacteriana ou retenção de placenta, mas não há alterações estruturais na parede dos cascos (Tadros e Frank, 2013).

O tratamento de equinos com SME portadores de laminite endocrinopática precisa associar perda de peso e conforto aos cascos do paciente. Dessa forma, o tratamento dos pacientes envolveu dieta com restrição de energia e suspensão de exercícios, visto que a atividade atlética poderia prejudicar a interface lamelar do casco (Johnson et al., 2010). O repouso dos animais ocorreu em baia forrada com tapetes de borracha e cama de maravalha para priorizar o bem-estar animal. Nos casos atendidos, a dieta preconizada foi baseada no fornecimento de feno na proporção de $1 \%$ a $1,5 \%$ do peso corporal apresentado pelo paciente, sendo que Frank (2010) indica que o animal receba feno em quantidade equivalente a $1,5 \%$ do peso corporal ideal.

O uso de fenilbutazona ou flunixina meglumina, ambos AINEs não seletivos para $\mathrm{COX} 2$, foi adequado para promover analgesia e reduzir a claudicação dos pacientes, pois tais fármacos são eficientes analgésicos na maioria dos casos de laminite (Eps, 2010). Os AINEs, preferencialmente COX2 e os seletivos COX2, como é o caso do meloxicam e do firocoxibe, respectivamente, têm menor potencial analgésico em comparação aos AINEs não seletivos para tratar casos agudos, mas, ainda assim, são adequados para controlar a dor de equinos com laminite crônica e geram significativamente menos efeitos colaterais (Eps, 2010). Assim, o Equino 3 beneficiou-se ao receber firocoxibe, uma vez que o tratamento pôde ser prolongado, e não foi necessário fazer uso injetável do medicamento.

A acepromazina na dose de 0,01-0,066mg/kg melhora o fluxo sanguíneo digital de equinos sadios em estação (Stashak, 2006). Nos pacientes relatados, empregou-se $0,01 \mathrm{mg} / \mathrm{kg}$ de acepromazina pela via intramuscular, a cada 12 horas, o que contribuiu para a diminuição da intensidade do pulso digital.
A biotina utilizada no Equino 1 tratou a fragilidade do casco, visto que esse suplemento melhora a qualidade do casco e o crescimento da muralha, o que beneficia a recuperação de equinos com laminite crônica, apesar de ser pouco efetivo na doença aguda (Stashak, 2006).

O casqueamento regular melhorou o apoio, aumentou o conforto e reduziu as comorbidades podais dos pacientes, uma vez que o casqueamento em equinos com laminite visa equilibrar o apoio, diminuir as forças de sustentação sobre a parede dorsal do casco e a tensão sobre o tendão flexor digital profundo, bem como manter a saúde dos cascos, evitando que outras doenças podais possam agravar a claudicação (Stashak, 2006).

\section{CONCLUSÕES}

O tratamento anti-inflamatório, em conjunto com o manejo específico da laminite, foi eficaz, sendo a restrição alimentar parte importante na terapia.

\section{REFERÊNCIAS}

EPS, A.W.V. Acute laminitis: medical and supportive therapy. Vet. Clin. Equine, v.26, p.103-114, 2010.

FRANK, N.; GEOR, R.J.; BAILEY, S.R. et al. Equine metabolic syndrome. J. Vet. Intern. Med., v.24, p.467-475, 2010.

HENNEKE, D.R.; POTTER, G.D.; KREIDER, J.L. Body condition during pregnancy and lactation and reproductive efficiency of mares. Theriogenology, v.21, p.897-909, 1983.

JOHNSON, P.J.; WIEDMEYER, C.E.; LACARRUBBA, A. et al. Laminitis and the equine metabolic syndrome. Vet. Clin. Equine, v.26, p.239-255, 2010.

MENZIES-GOW, N. Diagnosing and treating laminitis in horses. Vet. Rec., v.183, p.505-506, 2018.

OBEL, N. Studies on the histopathology of acute laminitis. Vet. Stockholm, v.1, p.1-50, 1948.

PAZ, C.F.R.; PAGANELA, J.C.; SANTOS, C.A. et al. Relação entre obesidade, insulina plasmática e posicionamento da falange distal em equinos da raça Crioula. Arq. Bras. Med. Vet. Zootec., v.65, p.1699-1705, 2013. 
POLLITT, C.C. Equine laminitis: a revised pathophysiology. AM. ASSOC. Equine Proc., v.45, p.188-192, 1999.

SILVA, G.B.; CÔRTEII, F.D.; BRASSIL, K.E. et al. Laminite crônica em equídeos da raça Crioula: características clínicas e radiográficas. Ciênc. Rural, v.43, p.2025-2030, 2013.

STASHAK, T.S. Claudicação em equinos segundo Adams. 5.ed. São Paulo: Roca, 2006. p.100, 611-613.
STICK, J.A.; JANN, H.W.; SCOTT, E.A.; ROBINSON, N.E. Pedal bone rotation as a prognostic sign in laminitis of horses. J. Am. Vet. Med. Assoc., v.180, p.251-253, 1982.

TADROS, E.M.; FRANK, N. Endocrine disorders and laminitis. Equine Vet. Educ., v.25, p.152-162, 2013. 